\title{
Genetic association study of CYP1A1 polymorphisms identifies risk haplotypes in nonsmall cell lung cancer
}

\author{
C.M. Wright*,\#,ף, J.E. Larsen*,\#, , M.L. Colosimo*, J.J. Barr*, L. Chen*, \\ R.E. McLachlan*, I.A. Yang*,\#, R.V. Bowman*, and K.M. Fong*
}

ABSTRACT: Lung cancer remains a leading cause of disease globally, with smoking being the largest single cause. Phase I enzymes, including cytochrome $P_{450}$, family 1 , subfamily $A$, polypeptide 1 (CYP1A1), are involved in the activation of carcinogens, such as polycyclic aromatic hydrocarbons, to reactive intermediates that are capable of binding covalently to DNA to form DNA adducts, potentially initiating the carcinogenic process. The aim of the present study was to investigate the association of CYP1A1 gene polymorphisms and haplotypes with lung cancer risk.

A case-control study was carried out on 1,040 nonsmall cell lung cancer (NSCLC) cases and 784 controls to investigate three CYP1A1 variants, CYP1A1*2A (rs4646903; thymidine to cytosine substitution at nucleotide $3801(3801 T>C)$ ), CYP1A1*2C (rs1048943; 2455A $>$ G; substitution of isoleucine 462 with valine (exon 7)) and CYP1A1*4 (rs1799814; 2453C $>A$; substitution of threonine 461 with asparagine (exon 7)) using PCR restriction fragment length polymorphism methods.

The CYP1A1*2A and CYP1A1*2C variants were significantly over-represented in NSCLC cases compared with controls, whereas the CYP1A1*4 variant was under-represented. CYP1A1 haplotypes (in allele order CYP1A1*4, CYP1A1*2C, CYP1A1*2A) CGC and CGT were associated with an increased risk of lung cancer, whereas AAT was associated with decreased lung cancer risk in this population.

The present study has identified risk haplotypes for CYP1A1 in NSCLC and confirmed that CYP1A1 polymorphisms are a minor risk factor for NSCLC.

KEYWORDS: Cytochrome $P_{450}$, cytochrome $P_{450} 1 A 1$ gene, haplotype, lung cancer, nonsmall cell lung cancer

ung cancer is a leading cause of cancer death internationally, with smoking being the largest single cause. Smoking is responsible for $85-90 \%$ of lung cancers [1], yet $<20 \%$ of lifelong smokers develop lung cancer, suggesting that other factors, including genetics, may play a role [1]. Phase I enzymes (mainly cytochrome $\mathrm{P}_{450}$ ) metabolically activate carcinogens, such as polycyclic aromatic hydrocarbons and $N$-nitrosamines, to reactive intermediates [2]. These intermediates are capable of binding covalently to DNA to form DNA adducts, potentially initiating the carcinogenic process. Two functionally important nonsynonymous polymorphisms have been described for the cytochrome $\mathrm{P}_{450}$, family 1 , subfamily A, polypeptide 1 (CYP1A1) gene, a base substitution at codon 462 in exon 7 , resulting in substitution of isoleucine with valine (Ile462Val (exon 7))
(CYP1A1*2C; National Center for Biotechnology Information single nucleotide polymorphism (SNP) identifier rs1048943; adenine (A) to guanine (G) substitution at nucleotide 2455 $(2455 \mathrm{~A}>\mathrm{G}))$ and a point mutation (thymine $(\mathrm{T})$ to cytosine $(\mathrm{C})$ ) at the MspI site in the $3^{\prime}$ untranslated region $\left(C Y P 1 A 1^{*} 2 A\right.$; rs4646903; $3801 \mathrm{~T}>$ C) $[3,4]$. A third polymorphism, substituting threonine (Thr) for asparagine (Asn) (CYP1A1*4; rs1799814; 2453C>A; Thr461Asn (exon 7)), has also been reported two bases upstream of $C Y P 1 A 1^{*} 2 C$; however, its functional effects remain to be fully elucidated.

Ethnic differences in the distribution of the CYP1A1*2C and CYPA1*2A genotypes have been reported in lung cancer subjects, with few reports available for $C Y P 1 A 1^{*} 4$. An over-representation of the Val allele $\left(C Y P 1 A 1^{*} 2 C\right)$ among lung cancer

\section{AFFILIATIONS}

*Dept of Thoracic Medicine, The

Prince Charles Hospital, and

${ }^{\#}$ School of Medicine, The University of Queensland, Brisbane, Australia.

"Both authors contributed equally to this study.

CORRESPONDENCE

C.M. Wright

Room 2

Level 1 Clinical Sciences Building

The Prince Charles Hospital

Rode Road

Chermside

QLD 4032

Australia

E-mail: c.wright@uq.edu.au

Received:

Aug 062008

Accepted after revision:

July 052009

First published online:

July 162009 
cases has been reported in Asian and Caucasian populations [5-7]. Although relatively frequent in Asian populations (0.180.25) $[8,9]$, the Val allele is quite rare in Caucasian control populations, occurring in $\sim 7-13 \%$ of people $[7,10]$. In a previous study, no interactive effects were shown between CYP1A1*2C and polymorphisms of the phase II enzyme genes encoding glutathione S-transferase pi 1 (GSTP1), theta 1 (GSTT1) or mu 1 (GSTM1). However, risk genotypes of the myeloperoxidase gene $(M P O)$ and $C Y P 1 A 1^{*} 2 C$ interacted to increase the overall risk of nonsmall cell lung cancer (NSCLC) (odds ratio (OR) 2.88; 95\% confidence interval (CI) 1.70-5.00; $\mathrm{p}=0.001$ ) [11]. Similarly, the $C Y P 1 A 1^{*} 2 A$ variant has been strongly associated with increased lung cancer risk in Asian populations, especially in relation to tobacco smoking and in combination with polymorphisms of the phase II enzyme genes GSTM1 and GSTT1 [12, 13]. In contrast, studies in Caucasian populations have not clearly established an association between CYP1A1 polymorphisms and increased lung cancer risk [14-19]. Relatively frequent incidences of CYP1A1*2A variants have been reported in Asian (39\%) [20] and Caucasian control populations (18-23\%) [14, 16, 21]. Conversely, few studies have reported population frequencies for CYP1A1*4 in lung cancer subjects. CASCORBI et al. [6] reported variant allele frequencies of $3 \%$ in Caucasian lung cancer cases and controls. In contrast, SoNG et al. [13] detected no polymorphic sites in an Asian population, supporting similar polymorphism studies of Japanese liver subjects [10]. Similar frequencies (2-3\%) have been observed in healthy Caucasian populations [22, 23]. A recent pooled analysis of HuNG et al. [24] reported an increase in lung cancer susceptibility by more than two-fold for CYP1A1*2A and CYP1A1*2C in nonsmokers. The authors suggested these polymorphisms may be implicated in lung carcinogenesis at low levels of tobacco exposure, possibly in combination with phase II enzymes, including GSTM1 [24], although this has not been replicated by other groups [21].

Many studies investigating the relationships between CYP1A1 polymorphisms and lung cancer have been limited by small sample numbers, leading to a lack of statistical power. Pooled analyses to increase sample size have tried to address this issue. Conflicting results between groups may be due to population differences (i.e. ethnicity) or failure to control for other potential confounders, including age and sex. CYP1A1 haplotype studies have potential in determining whether combinations of CYP1A1 (CYP1A1*2A, CYP1A1*2C, CYP1A1*4) together confer a greater risk of lung cancer than single polymorphisms. One recent study of 200 case-matched controls from an Indian population, showed that only one haplotype, CGC (3801C, 2455G, 2453C), was significantly associated with increased lung cancer risk, although the study was limited by its small sample numbers [3]. Few CYP1A1 haplotype association studies have been conducted in a Caucasian population. A study of HAN et al. [25] investigated the genotype frequencies of 13 SNPs found in the promoter region of CYP1A1 in 21 Caucasian individuals. Subsequent functional studies identified two CYP1A1 haplotypes $(2923 \mathrm{C}>\mathrm{T}, 2875 \mathrm{G}>\mathrm{A}, \quad 3777 \mathrm{~T}>\mathrm{G}$; and $2923 \mathrm{C}>\mathrm{T}, \quad 3777 \mathrm{~T}>\mathrm{G}, \quad 4553 \mathrm{G}>\mathrm{A}$ ) demonstrating moderate increases in basal activity compared with the wild-type CYP1A1 constructs ( 1.38 and 1.50 , respectively; $\mathrm{p}<0.05)$. These were considered unlikely to be of functional significance considering the magnitude of difference in CYP1A1 expression in response to benzo[a]pyrene and cigarette smoke extract [25].

In order to further investigate the role of $C Y P 1 A 1$ polymorphism variation $\left(C Y P 1 A 1^{*} 2 C, C Y P 1 A 1^{*} 2 A\right.$ and $\left.C Y P 1 A 1^{*} 4\right)$ in lung cancer risk, CYP1A1 haplotype analyses were performed in a large sample of Australian lung cancer cases with the aim of identifying risk-modifying CYP1A1 haplotypes.

\section{MATERIALS AND METHODS \\ Study population}

The present study population has been described previously $[11,26]$. Cases were subjects with confirmed primary lung cancer treated at The Prince Charles Hospital (Brisbane, Australia) during 1980-2007 $(n=1,040)$. Controls consisted of subjects with chronic obstructive pulmonary disease (COPD) treated at the same hospital $(n=506)$ or healthy smokers $(n=278)$ who, at the time of recruitment to the study, did not have doctor-diagnosed lung cancer. The ethnicity of the study population was $>99 \%$ Caucasian. The study was approved by the Ethics Committee at The Prince Charles Hospital. Cases and controls gave informed written consent for use of resected lung tissue or peripheral blood. The demographics of cases and controls were checked by a research nurse or the treating physician against patient medical charts or the hospital lung cancer database (table 1).

\section{Sample preparation and genotyping}

DNA from cases diagnosed with NSCLC was extracted from peripheral blood or resected cryopreserved normal lung tissue as described previously [26]. Cases and controls from a previous study of $C Y P 1 A 1^{*} 2 C$ were included $[11,26]$. DNA from control subjects was extracted from peripheral blood. In 592 cases, DNA was extracted from more than one source (blood lymphocytes and fresh frozen normal lung tissue). In these cases, both sources were genotyped, with identical results in all cases, reinforcing the reproducibility of the methods. PCR-based restriction fragment length polymorphism (RFLP) methods were used to analyse CYP1A1*2A [27] and CYP1A1*4 [6] polymorphisms. Approximately $10 \%$ of the samples were randomly selected for repeat genotyping by PCR-RFLP in order to test reproducibility.

\section{DNA sequencing and single-base extension genotyping}

In order to confirm the accuracy of the PCR-RFLP methods, two representative samples per genotype were confirmed by DNA sequencing for each polymorphism. Samples were purified using the Wizard PCR Cleanup and Gel extraction kit (Promega, Madison, WI, USA) and sequenced at the Australian Genome Research Facility (AGRF, Brisbane, Australia) for DNA sequencing using BigDye Terminator V3.1 chemistry (Applied Biosystems, Foster City, CA, USA). The sequencing primers were identical to those used in PCR amplification. Sequences were visualised using Chromas V1.4 (C. McCarthy, School of Biomolecular and Physical Sciences, Griffith University, Brisbane, Australia).

In addition, an independent genotyping method, iPLEX singlebase extension (Sequenom, San Diego, CA, USA), was also used to genotype a subset $(89 \%)$ of subjects genotyped by PCR-RFLP methods. All experiments were performed by the AGRF. Briefly, 


\begin{tabular}{|c|c|c|c|}
\hline \multirow[t]{2}{*}{ TABLE 1} & \multicolumn{3}{|c|}{$\begin{array}{l}\text { Demographics of cases and controls using the } \\
\text { PCR restriction fragment length polymorphism } \\
\text { method }\end{array}$} \\
\hline & NSCLC & Controls ${ }^{\#}$ & $p$-value ${ }^{*}$ \\
\hline Subjects $\mathrm{n}$ & 1040 & 784 & \\
\hline \multicolumn{4}{|l|}{ Sex } \\
\hline Male & $710(68)$ & $531(68)$ & 0.807 \\
\hline Female & 330 (32) & 253 (32) & \\
\hline Age yrs & $65.7 \pm 10(32-92)$ & $63.1 \pm 12.7$ (19-93) & $<0.0001$ \\
\hline $\begin{array}{l}\text { Smoking history } \\
\text { pack-yrs }^{+}\end{array}$ & $48 \pm 35.1(0-270)$ & $49.1 \pm 33.5(0-246)$ & 0.466 \\
\hline \multicolumn{4}{|l|}{ Histology } \\
\hline$A C$ & 407 (39) & & \\
\hline SCC & $373(36)$ & & \\
\hline Other & $260(25)$ & & \\
\hline \multicolumn{4}{|l|}{ Tumour stage } \\
\hline । & $422(41)$ & & \\
\hline ॥ & $202(19)$ & & \\
\hline III & $231(22)$ & & \\
\hline IV & $113(11)$ & & \\
\hline Unknown & $72(7)$ & & \\
\hline
\end{tabular}

Data are presented as $n(\%)$ or mean \pm SD (range) unless otherwise indicated. NSCLC: nonsmall cell lung cancer; AC: adenocarcinoma; SCC: squamous cell carcinoma. ${ }^{\#}$ : subjects with chronic obstructive pulmonary disease combined with healthy smokers; ": Chi-squared test or t-test, cases versus controls, with level of significance $(\alpha)$ considered to be 0.05 and significant values shown in bold type; ${ }^{+}$: a measure of cumulative smoking exposure, defined as the mean number of packs ( 20 cigarettes. pack $^{-1}$ ) of cigarettes smoked daily multiplied by the number of years of smoking.

genomic DNA was amplified using primers with 10-mer tags, designed to amplify a 75-150-bp amplicon. Following this, shrimp alkaline phosphatase was added to each reaction in order to remove any unincorporated deoxyribonucleoside triphosphates (dNTPs) by cleavage of phosphates from dNTP groups. An iPLEX reaction master mix consisting of primer, enzyme, buffer and mass-modified nucleotides was then added and the samples placed in a thermocycler in order to permit addition of nucleotides to the polymorphic site, producing allele-specific base extension products of differing sizes. Products were then run on a matrix-assisted laser desorption/ionisation time-offlight mass spectrometer in order to determine product size.

\section{Statistical analysis}

Distributions of genotypes and demographic variables were compared between cases and controls using Chi-squared tests for categorical outcome variables and two-sided unpaired t-tests for continuous outcome variables. ORs and 95\% CIs were estimated in order to measure the association between lung cancer and genotype/haplotype frequency. Standard Chisquared statistics were used to determine whether or not the three CYP1A1 variants were in Hardy-Weinberg equilibrium (HWE). All statistical analyses for genotypes were performed using the SPSS software package (Version 13.0 for Windows; SPSS, Inc., Chicago, IL, USA). Haplotype analyses (haplotype frequency estimates and linkage disequilibrium) were carried out using Haploview linkage software (Version 4.0) [28]. A p-value of $<0.05$ (two-tailed) was considered significant. Power calculations to evaluate ability to detect associations between NSCLC and CYP1A1 variants among the present study population were determined using an $\alpha$ of 0.05 and $80 \%$ power. The present study had the power to detect ORs of 1.39 , 1.48 and 1.69 for the variant alleles of CYP1A1*2A, CYP $1 A 1^{*} 4$ and $C Y P 1 A 1^{*} 2 C$, respectively, with $80 \%$ confidence at an $\alpha$ of 0.05 .

\section{RESULTS \\ Participant characteristics}

In order to ensure that observed effects between cases and controls were due to genotype frequency and not other potential confounding factors, such as age, smoking history or sex, mean and frequency distributions were compared between cases and controls (table 1). Mean age differed significantly between cases and controls $(p<0.001)$, with controls being slightly younger. In contrast, there were no significant differences in sex distribution $(p=0.81)$ or smoking history in pack-yrs $(\mathrm{p}=0.47)$, excluding these factors as potential study confounders. The majority of NSCLC cases were adenocarcinoma or squamous cell carcinoma histological subtypes (39 and $36 \%$, respectively).

In order to confirm the validity of combining COPD subjects and healthy smokers to form one control group, as done previously [26], Chi-squared tests were used to investigate whether or not there were significant differences in genotype frequency between the two groups. No significant difference in variant frequency was observed between COPD and healthy smokers ( $p>0.05$; data not shown), validating the decision to combine these two subgroups.

\section{PCR restriction fragment length polymorphism}

Genotype frequencies for all three variants, determined by PCR-RFLP methods, are detailed in table 2. Variant allele frequencies were low in the control population for all of the SNPs. The minor allele frequencies for cases and controls combined were $4.6,4.5$ and $11.4 \%$ for $C Y P 1 A 1^{*} 4, C Y P 1 A 1^{*} 2 C$ and $C Y P 1 A 1^{*} 2 A$, respectively. Since less than five samples were classified as homozygous for the variant allele in either cases or controls for CYP1A1*2C or CYP1A1*4, homozygous variants were combined with heterozygous genotypes for statistical analysis (table 3 ). In the present study, genotypes containing the variant allele of $C Y P 1 A 1^{*} 2 A$ occurred in $17.6 \%$ of the control population, CYP1A1*4 in $11 \%$ and $C Y P 1 A 1^{*} 2 C$ in $5.5 \%$. These values were in relative agreement with previous studies of Caucasian populations (CYP1A1*2A 18-23\%; CYP1A1*4 2-3\% and CYP1A1*2C 9-10\%) [14, 16, 21-23]. In the present study population, the genotype frequencies of CYP1A1*2A and CYP1A1*4 were in HWE for cases and controls; however, CYP $1 A 1^{*} 2 C$ was not (table 4). Sanger DNA sequencing of two samples per genotype was performed for each polymorphism in order to confirm the accuracy of the PCR-RFLP assays.

From the PCR-RFLP data, it was observed that subjects carrying the CYP1A1*2A variant were over-represented in NSCLC cases compared with controls (TT versus CT/CC; OR 1.43 (95\% CI 1.35-1.51); $\mathrm{p}=0.003)$. A decreased risk of NSCLC was also observed for those with the CYP1A1*4 variant (CC 
TABLE 2 Distribution of cytochrome $\mathrm{P}_{450} 1 \mathrm{~A} 1$ (CYP1A1) genotypes amongst nonsmall cell lung cancer subjects and controls ${ }^{\#}$

\begin{tabular}{|c|c|c|c|c|c|c|c|c|}
\hline & \multicolumn{2}{|c|}{ Cases } & \multicolumn{2}{|c|}{ Controls } & \multicolumn{2}{|r|}{ iPLEX } & \multicolumn{2}{|c|}{ p-value ${ }^{+}$} \\
\hline & PCR-RFLP & iPLEX & PCR-RFLP & iPLEX & Samples failed & Samples not re-genotyped & RFLP & iPLEX \\
\hline \multicolumn{9}{|c|}{ CYP1A1*2C } \\
\hline $\mathrm{AA}$ & 929 (89.3) & 827 (89.9) & $741(94.5)$ & $634(94.2)$ & $53(3.2)$ & $162(9.7)$ & $<0.001$ & 0.008 \\
\hline$A G$ & $103(9.9)$ & $89(9.7)$ & $40(5.1)$ & $37(5.5)$ & $2(1.4)$ & $11(7.7)$ & & \\
\hline \multicolumn{9}{|c|}{ CYP1A1*2A } \\
\hline $\mathrm{TT}$ & 797 (76.6) & $675(77.4)$ & $646(82.4)$ & $541(82.3)$ & $67(4.6)$ & $150(10.4)$ & 0.008 & 0.008 \\
\hline CT & $219(21.1)$ & 197 (22.6) & $128(16.3)$ & $116(17.7)$ & $20(5.8)$ & $21(6.1)$ & & \\
\hline $\mathrm{CC}$ & $24(2.3)$ & $24(2.7)$ & $10(1.3)$ & $8(1.2)$ & $1(2.9)$ & $3(8.8)$ & & \\
\hline Total $n$ & 1040 & 896 & 784 & 665 & 88 & 174 & & \\
\hline \multicolumn{9}{|c|}{ CYP1A1*4 } \\
\hline Total $n$ & 1040 & 920 & 784 & 672 & 58 & 173 & & \\
\hline
\end{tabular}

Data are presented as $n$ (\%) unless otherwise indicated. RFLP: restriction fragment length polymorphism; A: adenine; G: guanine; T: thymidine; C: cytosine. ${ }^{*}$ using PCRRFLP and Sequenom iPLEX (Sequenom, San Diego, CA, USA) methods; ": subjects with chronic obstructive pulmonary disease combined with healthy smokers; ${ }^{+}$: Chisquared test or t-test, cases versus controls, with level of significance $(\alpha)$ considered to be 0.05 and significant values shown in bold type.

versus CA/AA; OR $0.64(95 \%$ CI $0.62-0.64) ; p=0.005)$. The wild-type genotype (CC) was slightly over-represented in cases versus controls (92.8 versus $89 \%$ ). Data from a previous study on $C Y P 1 A 1^{*} 2 C$ were included in the present study. The variant allele was associated with a greater than two-fold increase in the risk of lung cancer.

In order to investigate associations between the three polymorphisms, haplotype analyses were performed to determine whether combinations of polymorphisms conferred a greater lung cancer risk. Five possible haplotypes were identified amongst cases and the larger control group, where the CAT haplotype was considered to be the wild-type (in allele order CYP1A1*4, CYP1A1*2C, CYP1A1*2A). The frequencies of CAT, CAC, AAT, CGC and CGT are presented in table 3. Two haplotypes were significantly over-represented in NSCLC subjects versus controls, CGC (4.1 versus $2.4 \%$; $=0.0038$; OR 1.76 (95\% CI 1.19-2.60)) and CGT (1.4 versus $0.4 \% ; \mathrm{p}=0.0026$; OR 3.26 (95\% CI 1.46-7.28)). Conversely, AAT was significantly under-represented in cases versus controls (3.4 versus $5.4 \%$; $\mathrm{p}=0.0014$; OR $0.62(95 \%$ CI $0.45-0.86))$. CAC showed a general increase in lung cancer risk, although this did not reach significance (8.5 versus $6.9 \%$; $\mathrm{p}=0.0747$; OR 1.25 (95\% CI 0.98 1.60)). It was found that $C Y P 1 A 1^{*} 2 A$ and $C Y P 1 A 1 * 2 C$ were in linkage disequilibrium (normalised disequilibrium constant (D') 0.749 ; logarithm of odds (logit; LOD) $66.5 ; \mathrm{r}^{2}=0.207 ; 95 \%$ CI $0.67-0.81)$ but that CYP1A1*4 and CYP1A1*2C (D' 0.01; LOD $0 ; \mathrm{r}^{2}=0 ; 95 \%$ CI $\left.0.01-0.696\right)$ and $C Y P 1 A 1^{*} 4$ and $C Y P 1 A 1 * 2 A$ were not ( $\mathrm{D}^{\prime} 0.582$; LOD $1.21 ; \mathrm{r}^{2}=0.0020 ; 95 \%$ CI $0.17-0.82$ ).

\section{Single-base extension genotyping}

As a secondary method of confirming PCR accuracy and to address the issue of Hardy-Weinberg disequilibrium, singlebase extension genotyping methods (iPLEX) were used to genotype cases and controls. It was possible to successfully regenotype $89 \%$ of cases and controls with iPLEX, with failure rates of 3-5\% for the three genotypes. Tables 2 and 3 illustrate the sample numbers and genotype and haplotype frequencies observed using iPLEX. All variants were in HWE for the control population (table 4), with two of three variants in HWE for cases (table 4).

Similar genotype frequencies were observed for $C Y P 1 A 1^{*} 2 C$, CYP1A1*4 and CYP1A1*2A, although relatively fewer variant homozygotes were observed for CYP1A1*4 (five for PCR-RFLP versus none for iPLEX in controls). Observed associations between CYP1A1 polymorphisms and lung cancer risk remained consistent, with subjects carrying the $C Y P 1 A 1 * 2 A$ variant over-represented among NSCLC cases compared to controls (TT versus CT/CC; OR 1.43 (95\% CI 1.35-1.52); $\mathrm{p}=0.005)$, and carriers of the $C Y P 1 A 1^{*} 4$ variant underrepresented in NSCLC cases (CC versus CA/AA; OR 0.66 (95\% CI 0.66-0.67); $\mathrm{p}=0.026)$. Carriers of the $C Y P 1 A 1^{*} 2 C$ variant were also associated with elevated lung cancer risk (AA versus AG/GG; OR 1.83 (95\% CI 1.57-2.12); $\mathrm{p}=0.002$ ), consistent with previous findings. Although the findings from PCR-RFLP remained consistent, a decrease in significance was observed, possibly due to the small decrease in sample number genotyped by iPLEX.

In order to ensure that the haplotype findings from PCR-RFLP remained consistent, new haplotype analyses were also performed on the iPLEX data. Only samples with iPLEX genotype data for all three polymorphisms were included in these analyses. Four possible haplotypes (CAT, CAC, CGC and AAT) were observed amongst cases and controls (in allele order CYP1A1*4, CYP1A1*2C,CYP1A1*2A; table 3), where CAT was considered to be the wild-type. No CGT haplotypes 


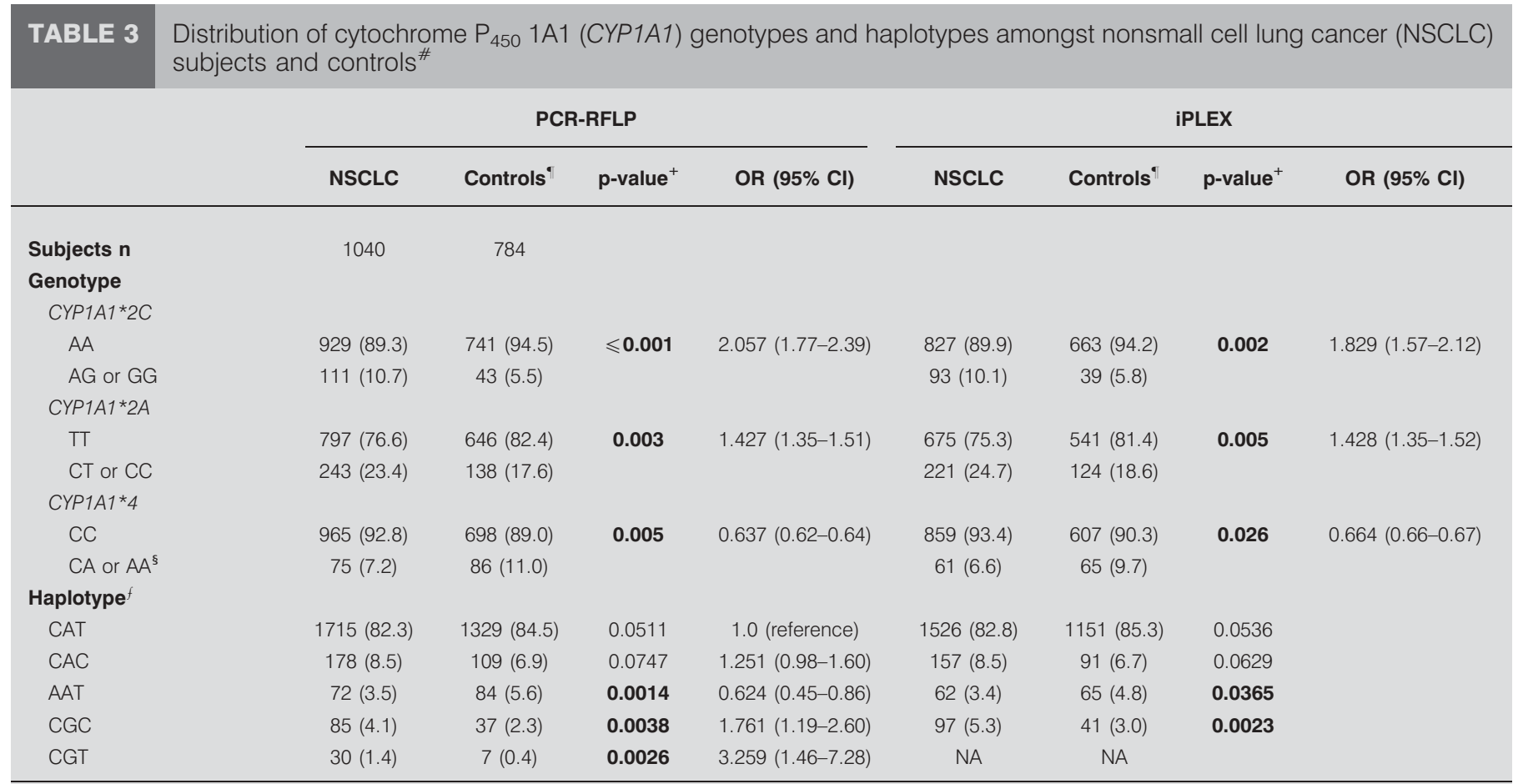

Data are presented as n (\%) unless otherwise indicated. RFLP: restriction fragment length polymorphism; OR: odds ratio; Cl: confidence interval; A: adenine; G: guanine; T: thymidine; C: cytosine; NA: not applicable. ${ }^{*}$ : using PCR-RFLP and Sequenom iPLEX (Sequenom, San Diego, CA, USA) methods; ": subjects with chronic obstructive pulmonary disease combined with healthy smokers; ${ }^{+}$: significant associations shown in bold type; ${ }^{\text {s: }}$ no AA genotypes observed in this cohort; ${ }^{f}$ : in allele order CYP1A1*4 (C to A substitution at nucleotide $2453(2453 \mathrm{C}>\mathrm{A})), \mathrm{CYP} 1 \mathrm{~A} 1{ }^{*} 2 \mathrm{C}(2455 \mathrm{~A}>\mathrm{G}), \mathrm{CYP} 1 \mathrm{~A} 1^{*} 2 \mathrm{~A}(3801 \mathrm{~T}>\mathrm{C})$.

were observed in this group. Two of these haplotypes were significantly associated with either increased (CGC; 5.3 versus $3.0 \% ; \mathrm{p}=0.0023$ ) or decreased lung cancer risk (AAT; 3.4 versus $4.8 \% ; \mathrm{p}=0.037)$, with CAC showing a trend towards increased lung cancer risk (8.5 versus $6.7 \% ; \mathrm{p}=0.063)$. These risk haplotypes were also identified in the present PCR-RFLP haplotype analyses. $C Y P 1 A 1^{*} 2 A$ and $C Y P 1 A 1^{*} 2 C$ remained in linkage disequilibrium ( $\mathrm{D}^{\prime} 1.0$; LOD 98.17; $\mathrm{r}^{2}=0.324 ; 95 \% \mathrm{CI}$ 0.97-1.00), with CYP1A1*4 and CYP1A1*2C (D' 0.991; LOD $0.67 ; \quad r^{2}=0.002 ; \quad 95 \%$ CI $\left.0.11-0.98\right)$ and CYP1A1*4 and CYP1A1*2A not exhibiting disequilibrium (D' 1.0; LOD 2.76;

TABLE 4 Hardy-Weinberg Chi-squared tests for nonsmall cell lung cancer (NSCLC) subjects and controls

\begin{tabular}{lccccc} 
Genotype & \multicolumn{2}{c}{ PCR-RFLP } & & \multicolumn{2}{c}{ iPLEX $^{\#}$} \\
\cline { 2 - 3 } \cline { 5 - 6 } & NSCLC & Controls & & NSCLC & Controls \\
\hline CYP1A1*2A & 0.16 & 0.45 & & 0.04 & 0.51 \\
CYP1A1*2C & 0.03 & 0.01 & & 0.34 & 0.07 \\
CYP1A1*4 & 0.48 & 0.27 & & 0.96 & 0.18 \\
\hline
\end{tabular}

p-values of $>0.05$ were considered to be consistent with Hardy-Weinberg equilibrium. RFLP: restriction fragment length polymorphism; CYP1A1: cytochrome $P_{450}$, family 1 , subfamily A, polypeptide 1 gene. ${ }^{\#}$ : Sequenom, San Diego, CA, USA. $r^{2}=0.006 ; 95 \%$ CI $\left.0.48-1.00\right)$, as observed in the PCR-RFLP findings.

\section{DISCUSSION}

It has previously been shown that carriers with the $C Y P 1 A 1^{*} 2 C$ $\mathrm{Val}$ allele (Ile/Val or Val/Val genotype) are significantly overrepresented in NSCLC subjects compared to controls (OR 1.9 (95\% CI 1.20-2.90); $\mathrm{p}=0.005)$, especially in females, those aged $<64$ yrs and those with $<46$ pack-yrs of tobacco exposure [26]. It has also previously been shown that the CYP1A1*2C variant, in combination with the $M P O$ risk allele, confers a significantly increased risk of NSCLC (OR 2.88 (95\% CI 1.70-5.00); p<0.0001) [11]. In the present report, we present the genotype and haplotype frequencies of two additional CYP1A1 polymorphisms in relation to lung cancer risk. These data indicate an association between $C Y P 1 A 1^{*} 2 A$ and lung cancer risk, and support a recent pooled analysis of 2,451 lung cancer subjects and 3,358 controls that showed a clear association between the homozygous CYP1A1*2A CC allele and lung cancer risk in Caucasians (ageand sex-adjusted OR 2.36 (95\% CI 1.16-4.81)) [15]. Although a meta-analysis of HoulsTON [29] provided little support for involvement in lung cancer risk of variations in CYP1A1, a recent review investigating the role of polymorphisms in candidate genes for 18 different cancer sites reported an increased risk of lung cancer for carriers of the CYP1A1*2A variant in Caucasian populations (OR $2.36(95 \% 1.16-4.81) ; \mathrm{p}=0.018)$ in addition to variants of CYP1A1*2C in Asian populations (OR 1.61 (95\% CI 1.24-2.08); $\mathrm{p}=0.0003$ ) [30]. Associations between $C Y P 1 A 1$ variations and lung cancer risk have also been observed in neversmokers with lung cancer $[24,31]$. 
CYP1A1*2A and CYP1A1*2C have been reported to be in linkage disequilibrium in Caucasian [18] and Asian populations [27], although not in people of African descent [32]. Linkage disequilibrium can be influenced by a variety of factors, including genetic linkage, recombination, mutation rates, random drift, nonrandom mating and population structure. In the present study, $C Y P 1 A 1^{*} 2 A$ and $C Y P 1 A 1^{*} 2 C$ were in linkage disequilibrium, confirming reports from previous studies [18]. Conversely, as in CASCORBI et al. [6], no evidence of linkage disequilibrium between $C Y P 1 A 1^{*} 2 C$ and $C Y P 1 A 1^{*} 4$ was observed. This may be due to the close proximity of the two variants (only one base pair separates the two polymorphic sites) decreasing the rate of recombination.

The function of CYP1A1*4 has not been clearly established, although it has been suggested that it shows the greatest enzymatic efficiency of all of the CYP1A1 polymorphisms [33]. In the present study, very few homozygous variants of CYP1A1*4 were identified among cases or controls, limiting any ability to draw strong conclusions for a Caucasian population. However, a general decrease in the frequency of heterozygotes was observed in this study population compared to controls. Others have reported no clear association between lung cancer risk and CYP1A1*4 [3].

Very few haplotype analyses studying the interactions between $C Y P 1 A 1^{*} 2 A, C Y P 1 A 1^{*} 2 C$ and $C Y P 1 A 1^{*} 4$ have been performed in populations of Caucasian lung cancer subjects. A recent pooled analysis studying interactions between $C Y P 1 A 1$ polymorphisms and GSTM1/GSTT1 in an Asian population identified the GT and AC haplotypes as being associated with lung cancer risk compared to AT (OR 3.41 (95\% CI 1.78-6.53) and 1.39 (1.12-1.71), respectively) [34]. Among the present population, the haplotypes CGC and CGT (in allele order CYP1A1*4, CYP1A1*2C, CYP1A1*2A) were associated with an increased risk of lung cancer, whereas AAT was associated with a decreased risk. In an Indian population, a four-fold increased lung cancer risk (hazard ratio 3.90 (95\% CI 1.005.10); $p=0.025)$ was shown for the CGC haplotype [3], suggesting that this haplotype may be an important risk factor for NSCLC. Although the CAC haplotype has been associated with higher enzymatic activity and decreased cancer risk in prostate cancer [35], SHAH et al. [3] showed a trend for increased lung cancer risk, although this did not reach significance. The differences observed may be due to tumour type, gene expression or functional role of CYP1A1. For example, in addition to detoxification of carcinogens, CYP1A1 also has a role in the oxidative metabolism of oestrogens [36], which have been implicated in the aetiology of prostate cancer $[37,38]$. CHANG et al. [35] suggested that, in prostate cancer, CYP1A1's role of oestrogen metabolism may be more important than that of carcinogen detoxification. Therefore, it is possible that the role of CYP1A1 differs between cancer types, resulting in haplotype associations that vary according to tumour origin.

Interpretation of the data from the present study are curtailed by the low frequency of some variant genotypes (for instance very few $C Y P 1 A 1 * 4$ AA genotypes were observed) limiting any ability to draw statistically valid conclusions. Subjects in the control group also have the potential to develop lung cancer in the future, a source of misclassification bias. One of the polymorphisms, $C Y P 1 A 1^{*} 2 C$ was not in HWE in either controls or cases using PCR-RFLP, but was in HWE using iPLEX. In addition, although $C Y P 1 A 1^{*} 2 A$ was in HWE using PCR-RFLP, it was not in HWE using iPLEX in NSCLC subjects. This may be due to chance (from the selection of a large subset of the original samples), decreased sensitivity of iPLEX due to differences in sample concentration $\left(10 \mathrm{ng} \cdot \mu \mathrm{L}^{-1}\right.$ for iPLEX versus $10-40 \mathrm{ng} \cdot \mu \mathrm{L}^{-1}$ for PCR-RFLP, as determined by gel estimation techniques), a nonrandom population (highly unlikely) or a possible association with NSCLC risk. In addition, $C Y P 1 A 1^{*} 4$ and $C Y P 1 A 1^{*} 2 C$ are separated by only one nucleotide on exon 7 , raising the possibility that misclassification could occur. In the present study, an attempt was made to overcome this by employing published assays using enzymes that selectively digest each polymorphism irrespective of the neighbouring polymorphism, thereby decreasing the risk of misclassification bias. Finally, the present study evaluated three commonly studied CYP1A1 variants; however, it is possible that other SNPs may be in linkage disequilibrium with these variants, and may also be instrumental in determining lung cancer risk.

Technical validation of the present PCR-RFLP method by iPLEX confirmed the observed associations for all of the CYP1A1 polymorphisms. Similar genotype frequencies were observed for $C Y P 1 A 1^{*} 2 C, C Y P 1 A 1^{*} 4$ and $C Y P 1 A 1^{*} 2 A$ in both case and control populations. Haplotype analyses also confirmed association of haplotypes AAT and CGC with decreased and increased lung cancer risk, respectively. No CGT haplotypes were observed in these analyses, and association with lung cancer risk could not be confirmed. Despite the significance of these results decreasing in the iPLEX cohort, it remained possible to confirm the observations obtained using PCR-RFLP, increasing our confidence in the reproducibility of genotyping techniques. Owing to the high reproducibility rate, we are confident that these associations are valid and support a role for CYP1A1 polymorphisms in altering lung cancer risk.

In conclusion, the results of the present study confirm that CYP1A1 polymorphisms are a minor risk factor for NSCLC. Although several studies have confirmed associations between these polymorphisms and lung cancer risk in the past, few have explored linkage effects between the three different CYP1A1 polymorphisms in a Caucasian population. To the present authors' knowledge, this is the first study to show that haplotypes CGC, CGT and AAT are strongly associated with lung cancer risk in Caucasians. Identification of these haplotypes may assist in risk stratification, early detection and improvement of current treatment options for subjects with lung cancer. Larger studies are required in order to explore these risk CYP1A1 haplotypes further.

\section{SUPPORT STATEMENT}

This study was supported by The Prince Charles Hospital Foundation (Brisbane), a Queensland Smart State (Brisbane) Fellowship (K.M. Fong), a National Health and Medical Research Council (NHMRC; Canberra) Practitioner Fellowship (K.M. Fong), a Queensland Cancer Fund (Brisbane) Clinical Research Fellowship (K.M. Fong) and an NHMRC Career Development Award (I.A. Yang) (all Australia). 


\section{STATEMENT OF INTEREST}

None declared.

\section{ACKNOWLEDGEMENTS}

The authors would like to thank A. Semmler (Thoracic Research Laboratory, Prince Charles Hospital, Chermside, Australia). The authors also wish to thank the physicians, surgeons and pathologists at The Prince Charles Hospital (Brisbane, Australia) who assisted with the project; the subjects and donors who participated in the study; L.H. Passmore for recruiting subjects and confirming clinical data; and technical staff for their assistance with DNA extractions.

\section{REFERENCES}

1 Shopland D, Eyre H, Pechacek T. Smoking-attributable cancer mortality in 1991: is lung cancer now the leading cause of death among smokers in the United States? J Natl Cancer Inst 1991; 83: 1142-1148.

2 Bouchardy C, Benhamou S, Jourenkova N, et al. Metabolic genetic polymorphisms and susceptibility to lung cancer. Lung Cancer 2001; 32: 109-112.

3 Shah PP, Singh AP, Singh M, et al. Interaction of cytochrome P4501A1 genotypes with other risk factors and susceptibility to lung cancer. Mutat Res 2008; 639: 1-10.

4 Kawajiri K, Eguchi H, Nakachi K, et al. Association of CYP1A1 germ line polymorphisms with mutations of the $p 53$ gene in lung cancer. Cancer Res 1996; 56: 72-76.

5 Yang XR, Wacholder S, Xu Z, et al. CYP1A1 and GSTM1 polymorphisms in relation to lung cancer risk in Chinese women. Cancer Lett 2004; 214: 197-204.

6 Cascorbi I, Brockmoller J, Roots I. A C4887A polymorphism in exon 7 of human CYP1A1: population frequency, mutation linkages, and impact on lung cancer susceptibility. Cancer Res 1996; 56: 4965-4969.

7 Sugimura H, Wakai K, Genka K, et al. Association of Ile462Val (exon 7) polymorphism of cytochrome P450 IA1 with lung cancer in the Asian population: further evidence from a case-control study in Okinawa. Cancer Epidemiol Biomarkers Prev 1998; 7: 413-417.

8 Chowbay B, Zhou S, Lee EJ. An interethnic comparison of polymorphisms of the genes encoding drug-metabolizing enzymes and drug transporters: experience in Singapore. Drug Metab Rev 2005; 37: 327-378.

9 Oyama T, Mitsudomi T, Kawamoto T, et al. Detection of CYP1A1 gene polymorphism using designed RFLP and distributions of CYP1A1 genotypes in Japanese. Int Arch Occup Environ Health 1995; 67: 253-256.

10 Inoue K, Asao T, Shimada T. Ethnic-related differences in the frequency distribution of genetic polymorphisms in the CYP1A1 and CYP1B1 genes in Japanese and Caucasian populations. Xenobiotica 2000; 30: 285-295.

11 Larsen JE, Colosimo ML, Yang IA, et al. CYP1A1 Ile462Val and MPO G-463A interact to increase risk of adenocarcinoma but not squamous cell carcinoma of the lung. Carcinogenesis 2006; 27: 525-532.

12 Sreeja L, Syamala V, Hariharan S, et al. Possible risk modification by CYP1A1, GSTM1 and GSTT1 gene polymorphisms in lung cancer susceptibility in a South Indian population. J Hum Genet 2005; 50: 618-627.

13 Song N, Tan W, Xing D, et al. CYP 1A1 polymorphism and risk of lung cancer in relation to tobacco smoking: a case-control study in China. Carcinogenesis 2001; 22: 11-16.

$14 \mathrm{Xu} \mathrm{X,} \mathrm{Kelsey} \mathrm{KT,} \mathrm{Wiencke} \mathrm{JK,} \mathrm{et} \mathrm{al.} \mathrm{Cytochrome} \mathrm{P450} \mathrm{CYP1A1}$ MspI polymorphism and lung cancer susceptibility. Cancer Epidemiol Biomarkers Prev 1996; 5: 687-692.

15 Vineis P, Veglia F, Benhamou S, et al. CYP1A1 $T^{3801}$ C polymorphism and lung cancer: a pooled analysis of 2,451 cases and 3,358 controls. Int J Cancer 2003; 104: 650-657.
16 Cote ML, Wenzlaff AS, Bock $\mathrm{CH}$, et al. Combinations of cytochrome P-450 genotypes and risk of early-onset lung cancer in Caucasians and African Americans: a population-based study. Lung Cancer 2007; 55: 255-262.

17 Tefre T, Ryberg D, Haugen A, et al. Human CYP1A1 (cytochrome P1450) gene: lack of association between the Msp I restriction fragment length polymorphism and incidence of lung cancer in a Norwegian population. Pharmacogenetics 1991; 1: 20-25.

18 Hirvonen A, Husgafvel-Pursiainen K, Karjalainen A, et al. Pointmutational MspI and Ile-Val polymorphisms closely linked in the CYP1A1 gene: lack of association with susceptibility to lung cancer in a Finnish study population. Cancer Epidemiol Biomarkers Prev 1992; 1: 485-489.

19 Jacquet M, Lambert V, Baudoux E, et al. Correlation between P450 CYP1A1 inducibility, MspI genotype and lung cancer incidence. Eur J Cancer 1996; 32A: 1701-1706.

20 Boyapati SM, Shu XO, Gao YT, et al. Polymorphisms in CYP1A1 and breast carcinoma risk in a population-based case-control study of Chinese women. Cancer 2005; 103: 2228-2235.

21 Wenzlaff AS, Cote ML, Bock $\mathrm{CH}$, et al. CYP1A1 and CYP1B1 polymorphisms and risk of lung cancer among never smokers: a population-based study. Carcinogenesis 2005; 26: 2207-2212.

22 Mrozikiewicz PM, Cascorbi I, Brockmöller J, et al. CYP1A1 mutations 4887A, 4889G, 5639C and 6235C in the Polish population and their allelic linkage, determined by peptide nucleic acidmediated PCR clamping. Pharmacogenetics 1997; 7: 303-307.

23 Bosch TM, Doodeman VD, Smits PH, et al. Pharmacogenetic screening for polymorphisms in drug-metabolizing enzymes and drug transporters in a Dutch population. Mol Diagn Ther 2006; 10: 175-185.

24 Hung RJ, Boffetta P, Brockmoller J, et al. CYP1A1 and GSTM1 genetic polymorphisms and lung cancer risk in Caucasian non-smokers: a pooled analysis. Carcinogenesis 2003; 24 875-882.

25 Han W, Pentecost BT, Spivack SD. Functional evaluation of novel single nucleotide polymorphisms and haplotypes in the promoter regions of CYP1B1 and CYP1A1 genes. Mol Carcinog 2003; 37: 158-169.

26 Larsen JE, Colosimo ML, Yang IA, et al. Risk of non-small cell lung cancer and the cytochrome P4501A1 Ile462Val polymorphism. Cancer Causes Control 2005; 16: 579-585.

27 Hayashi S, Watanabe J, Nakachi K, et al. Genetic linkage of lung cancer-associated Ms $\mathrm{I}$ polymorphisms with amino acid replacement in the heme binding region of the human cytochrome P450IA1 gene. J Biochem 1991; 110: 407-411.

28 Barrett JC, Fry B, Maller J, et al. Haploview: analysis and visualization of LD and haplotype maps. Bioinformatics 2005; 21 263-265.

29 Houlston RS. CYP1A1 polymorphisms and lung cancer risk: a meta-analysis. Pharmacogenetics 2000; 10: 105-114.

30 Dong LM, Potter JD, White E, et al. Genetic susceptibility to cancer: the role of polymorphisms in candidate genes. JAMA 2008; 299 2423-2436.

31 Taioli E, Gaspari L, Benhamou S, et al. Polymorphisms in CYP1A1, GSTM1, GSTT1 and lung cancer below the age of 45 years. Int Epidemiol 2003; 32: 60-63.

32 Taioli E, Crofts F, Trachman J, et al. Radical differences in CYP1A1 genotype and function. Toxicol Lett 1995; 77: 357-362.

33 Schwarz D, Kisselev P, Honeck H, et al. Co-expression of human cytochrome P4501A1 (CYP1A1) variants and human NADPHcytochrome $\mathrm{P} 450$ reductase in the baculovirus/insect cell system. Xenobiotica 2001; 31: 345-356.

34 Lee KM, Kang D, Clapper ML, et al. CYP1A1, GSTM1, and GSTT1 polymorphisms, smoking, and lung cancer risk in a pooled analysis among Asian populations. Cancer Epidemiol Biomarkers Prev 2008; 17: 1120-1126. 
35 Chang BL, Zheng SL, Isaacs SD, et al. Polymorphisms in the CYP1A1 gene are associated with prostate cancer risk. Int J Cancer 2003; 106: 375-378.

36 Kristensen VN, Kure EH, Erikstein B, et al. Genetic susceptibility and environmental estrogen-like compounds. Mutat Res 2001; 482: 77-82.
37 Wang Y, Sudilovsky D, Zhang B, et al. A human prostatic epithelial model of hormonal carcinogenesis. Cancer Res 2001; 61: 6064-6072.

38 Hsing AW. Hormones and prostate cancer: where do we go from here? J Natl Cancer Inst 1996; 88: 1093-1095. 